\title{
Morbidade hospitalar e sua relação com variáveis climáticas na Cidade de Manaus: casos e custo econômico
}

Hospital morbidity and its relationship to climate variables in the City of Manaus: cases and economic cost

Morbilidad hospitalaria y su relación con las variables climáticas en la Ciudad de Manaus: casos y coste económico

Recebido: 15/10/2021 | Revisado: 22/10/2021 | Aceito: 24/10/2021 | Publicado: 27/10/2021

Flávia Thais da Silva Lima ORCID: https://orcid.org/0000-0002-7050-6571

Centro Universitário Fametro, Brasil E-mail: flavia.thayss@gmail.com

Alexandra Amaro de Lima ORCID: https://orcid.org/0000-0003-3918-0013 Centro Universitário Fametro, Brasil alexandra.lima@fametro.edu.br

Leonara dos Santos Cordeiro ORCID: https://orcid.org/0000-0002-9051-5512 Centro Universitário Fametro, Brasil E-mail: leonaraptrkati@gmail.com

Renã Raio do Nascimento ORCID: https://orcid.org/0000-0002-2265-5986 Centro Universitário Fametro, Brasil E-mail: renaraio02@gmail.com

Marcelo Menezes

ORCID: https://orcid.org/0000-0002-2324-6260 Centro Universitário Fametro, Brasil E-mail: marcelonezes2013@gmail.com

\begin{abstract}
Resumo
Este estudo avaliou o impacto das variáveis climáticas (precipitação, temperatura e umidade relativa) na incidência das doenças respiratórias representadas pela CID-10, em Manaus-AM. O aumento gradativo da população mundial está diretamente relacionado ao crescimento na utilização dos recursos dos provenientes do meio ambiente natural. Como a desigualdade social e a poluição ambiental estão relacionadas às doenças respiratórias, é necessário criar ações públicas e privadas para controlar e monitorar o nível dos poluentes relacionados, a fim de encontrar alternativas para reduzir os efeitos nocivos à saúde da população. Este estudo tem como objetivo analisar o número de pessoas internadas por doenças respiratórias em Manaus/AM durante o período de 1998 a 2020 e a relação dessas doenças com dados atmosféricos. Os dados foram obtidos em hospitais públicos e privados cadastrados no Sistema Único de Saúde-SUS (DATASUS) por meio da ficha de internação (AIH). O índice de custo de recursos econômicos ao SUS, acompanha o total de casos, apresentando um total de $\mathrm{R} \$ 19.048,28$, relacionado as internações. Após a análise verificou-se uma forte relação das informações de morbidade com o registro de doenças respiratórios e os registros de umidade e temperatura na região.
\end{abstract}

Palavras-chave: Morbidade; Condições climáticas; Doenças respiratórias.

\begin{abstract}
This study evaluated the impact of climatic variables (precipitation, temperature and relative humidity) on the incidence of respiratory diseases represented by the ICD-10, in Manaus-AM. The gradual increase in the world population is directly related to the growth in the use of resources from the natural environment. As social inequality and environmental pollution are related to respiratory diseases, it is necessary to create public and private actions to control and monitor the level of related pollutants, in order to find alternatives to reduce the harmful effects on the population's health. This study aims to analyze the number of people hospitalized for respiratory diseases in Manaus/AM during the period from 1998 to 2020 and the relationship of these diseases with atmospheric data. Data were obtained from public and private hospitals registered in the Unified Health System-SUS (DATASUS) through the admission form (AIH). The cost index of economic resources to the SUS follows the total number of cases, presenting a total of R $\$ 19,048.28$, related to hospitalizations. After the analysis, there was a strong relationship between morbidity information and the registry of respiratory diseases and the humidity and temperature records in the region.
\end{abstract}


Keyword: Morbidity; Climatic conditions; Respiratory diseases.

\section{Resumen}

Este estudio evaluó el impacto de las variables climáticas (precipitación, temperatura y humedad relativa) sobre la incidencia de enfermedades respiratorias representadas por la CIE-10, en Manaus-AM. El aumento gradual de la población mundial está directamente relacionado con el crecimiento en el uso de los recursos del medio natural. Dado que la desigualdad social y la contaminación ambiental están relacionadas con las enfermedades respiratorias, es necesario crear acciones públicas y privadas para controlar y monitorear el nivel de contaminantes relacionados, con el fin de encontrar alternativas para reducir los efectos nocivos en la salud de la población. Este estudio tiene como objetivo analizar el número de personas hospitalizadas por enfermedades respiratorias en Manaus / AM durante el período de 1998 a 2020 y la relación de estas enfermedades con los datos atmosféricos. Los datos se obtuvieron de los hospitales públicos y privados registrados en el Sistema Único de Salud-SUS (DATASUS) a través del formulario de admisión (AIH). El índice de costo de los recursos económicos al SUS sigue el número total de casos, presentando un total de R \$19.048,28, relacionados con hospitalizaciones. Luego del análisis, hubo una fuerte relación entre la información de morbilidad y el registro de enfermedades respiratorias y los registros de humedad y temperatura en la región.

Palabras clave: Morbilidad; Condiciones climáticas; Enfermedades respiratorias.

\section{Introdução}

O aumento gradativo da população mundial está diretamente relacionado ao crescimento na utilização dos recursos dos provenientes do meio ambiente natural. Essa forte relação leva ao esgotamento e a degradação desses recursos, que podem afetar de forma significativa à perda de saúde e qualidade de vida da população (Moraes \& Jordão, 2002; Santana et. al., 2020), e ao mesmo tempo, a degradação do meio ambiente promove a alteração dos fatores ambientais. Alguns estudos como os de Ribeiro (2004); Bruzos et. al., (2011) mostraram que os fatores ambientais e a saúde apresentam uma forte interação. Paralelamente, Barcelos et. al., (2009) mostram que mudanças no uso da terra, flutuações da temperatura e umidade, mudanças no regime das chuvas, poluição atmosférica potencializam em vários níveis os efeitos das doenças, respiratórias, cardiovasculares.

Além da degradação, Barcellos \& Hacon (2009); Barcellos et. al., (2016) mostraram que as variáveis climáticas como temperatura, umidade relativa e os eventos extremos de chuva podem proporcionar a proliferação das doenças ocasionadas por vetores. Ainda relacionado aos vetores, Barcellos et. al., (2016) apresentou um panorama mundial das doenças originárias pelo aumento dos vetores, apontando os países posicionados na faixa tropical e subtropical como os mais afetados pela dengue, enquanto, nos países amazônicos e do Sudeste da Ásia a malária é classificada como problema de saúde pública. Segundo Radunz (2013), a poluição atmosférica é um dano causado por alterações e degradação ambientais, centros industriais e crescimento de veículos automotores. Visto que, surge provocando emissão de gases tóxicos e poluentes, gerando situações críticas para a saúde da população.

De acordo com a organização Pan-Americana da Saúde (2008), o primeiro relatório global sobre as mudanças climáticas e a saúde da população foi publicado pela Organização Mundial da Saúde (OMS) em 1990 (World Health Organization, 1990). Desde então, a OMS tem mostrado forte preocupação com o papel das mudanças climáticas interferindo no meio ambiente natural e urbano, e acima de tudo, na saúde humana.

Estudar os impactos das mudanças climáticas na saúde é um processo extremamente complexo, pois requer um grupo de pesquisadores multidisciplinares, nas áreas de saúde, climatologia, cientistas sociais, biólogos, físicos, químicos, epidemiologistas, etc, e desta forma, será possível obter uma abordagem abrangente na relação entre as sociedades, sistemas econômicos, biológicos, ecológicos e físicos e sua relação com as mudanças climáticas (Mcmichael, 2003).

Devido ao difícil acesso, estudos sobre a região muitas vezes se tornam inviável, principalmente no monitoramento das queimadas, mas por outro, o uso de observações de sensores remotos, podem mostrar até a intensa combustão da biomassa em áreas em torno de milhões de $\mathrm{km}^{2}$ (Freitas et al., 2005). Em relação ao desmatamento, a taxa média de desmatamento na década 1990 era de $17.000 \mathrm{~km}^{2}$ por ano, que aumentando para aproximadamente $25.000 \mathrm{~km} 2 \mathrm{em} 2002$ e 2003 (Instituto Nacional de Pesquisas Espaciais - INPE, 2008). Em um estudo durante o período de 2014 a 2015 em Porto Velho/RO, Torres \& Medeiros 
(2019) verificaram que a exposição diária da população as concentrações de $\mathrm{PM}_{2,5}$ e $\mathrm{CO}_{2}$ geradas pelas queimadas na região, aumentaram significativamente as internações devidas de patologias do aparelho respiratório.

Contudo, as queimadas não afetam somente a população próximo a fonte poluidora, Serra \& Mülfarth (2021) as queimadas na região centro-oeste, foram responsáveis pelo aumento significativo do número de internações por doenças do aparelho respiratório, principalmente com os idosos na região de Ribeirão Preto/SP, durante o mês de setembro. Esse mês do ano segundo é mais quente e seco nas regiões norte e centro-oeste, mês de maior ocorrência de $\mathrm{PM}_{2,5}, \mathrm{CO}_{2}$ e aerossóis de origem das queimadas na Amazônia e no Pantanal (Souza et al., 2021).

Por outro lado, as doenças cardiovasculares e das vias respiratórias podem ser potencializadas nos grandes centros urbanos pela poluição atmosférica, associadas muitas vezes as oscilações nos registros das variáveis meteorológicas. Segundo Dutra et. al., (2021) o setor de transporte é responsável por grande parte das emissões dos gases poluentes atmosféricos, que associados aos episódios de extremos climáticos, como secas e cheias extremas, conduzem a um aumento das patologias das vias respiratórias e cardiovasculares (Barcellos et. al., 2009; Anjos \& Ferreira, 2011).

Como condições atmosféricas afetam o transporte de microrganismos, assim como de poluentes oriundos de fontes fixas e móveis e a produção de pólen (Moreno, 2006). Para Paiva (2014) a poluição atmosférica é vista como um dos principais gatilhos que podem interferir na saúde e qualidade de vida, visto que afetam enormemente o bem-estar da população, causam prejuízos na economia, entre outros fatores, já que a maior parte das internações hospitalares nos grandes centros são advindos das doenças respiratórias.

De acordo os registros do IBGE e do DATASUS, a poluição do ar é um dos problemas sociais relacionados à saúde humana e ao ambiente político. Por meio de perdas econômicas, vários estudos têm sido realizados para avaliar a relação entre poluição do ar e morbidade, e para identificar as principais doenças e poluição. Como a desigualdade social e a poluição ambiental estão relacionadas às doenças respiratórias, é necessário criar ações públicas e privadas para controlar e monitorar o nível dos poluentes relacionados, a fim de encontrar alternativas para reduzir os efeitos nocivos à saúde da população.

Segundo a Organização Pan-Americana da Saúde, as doenças respiratórias são influenciadas por queimadas, pelos efeitos de inversões térmicas que concentram a poluição próximo a superfície, impactando diretamente a qualidade do ar nas áreas urbanas. Ao mesmo tempo, as mudanças climáticas podem interferir na saúde humana de forma direta como, as ondas de calor, furações, inundações, e indireta devido a alteração do homem no ecossistema onde podem aumentar os fatores prejudiciais à saúde.

As variáveis meteorológicas associadas à poluição atmosférica são responsáveis pelos atendimentos de emergência em hospitais, internações e aumento do consumo de medicamentos nos hospitais da rede pública e privada (IBGE 2021). O comportamento e a variabilidade dos elementos climáticos, como a temperatura e umidade, de acordo Pitton \& Domingos (2004) exerceram um aumento nos casos de pacientes hipertensivos com idades acima de 40 anos, na cidade de Presidente Prudente/SP. Enquanto, Aleixo \& Sant'Anna Neto (2014) verificaram que as variáveis climáticas como a temperatura e umidade podem contribuir para o aumento da pneumonia na cidade de Ribeirão Preto/SP. Já Kleerekoper et al., (2012) apontam o conforto térmico nas grandes cidades pode ser a causa das morbidades e enfermidades, enquanto para Mandú et. al., (2019) a umidade relativa foi a responsável pelo crescimento do número de hospitalizações dos moradores da cidade de Manaus/AM com mais de 60 anos.

Mudanças bruscas de variáveis meteorológicas com a umidade relativa e a temperatura, podem modificar a concentração dos poluentes presentem na atmosfera, ou até mesmo interagir com o poluente, como o caso das reações fotoquímicas geradas durante o processo das queimadas (Artaxo et. al., 2005), mas podem resultar no aumento das internações por doenças respiratórias (Andreão et al., 2019; Santos et al., 2021). Devido sua importância no cenário atual das mudanças climáticas, as variáveis meteorológicas e a incidência de doenças cardiovasculares foram igualmente analisadas em Magalhães et al., (2019). 
Os autores mostraram que além da temperatura e umidade, a precipitação foi uma das variáveis responsável pelo aumento das internações na cidade de Manaus/AM. Durante o período de 1998 a 2016 na cidade de Campina Grande/PB, Carvalho et al., (2021) mostraram que a temperatura, umidade relativa do ar e a precipitação foram as variáveis meteorológicas responsáveis pelo aumento dos casos de pneumonia, nos três meses de verão os registros de casos de pneumonia estavam em torno de 10.577, enquanto que os meses maio a agosto são em torno de 21.101 casos.

Como visto até o momento, o homem deve e pode desfrutar do meio em que vive, mas vimos que o uso incorreto pode desencadear uma série de danos sem precedente ao meio e ao homem. Contudo, o artigo 225 da Constituição Federal de 1998 estipula que "todos têm direito a desfrutar de um meio ambiente ecologicamente equilibrado. Este é o interesse comum das pessoas e é essencial para uma qualidade de vida saudável. O poder público e a comunidade têm o dever de defendê-lo e servir às gerações presentes e futuras. Este estudo tem como objetivo analisar a relação entre o número de internações por doenças respiratórias na cidade de Manaus (AM), durante o período de 1998 a 2020 e as variáveis meteorológicas.

\section{Metodologia}

Considerando a população residente em Manaus no período de 1998 a 2020, este estudo foi realizado a partir de séries históricas de dados de internações por doenças respiratórias (RD). Os dados vêm de formulários de admissão (AIH) de hospitais públicos e privados cadastrados no Sistema Único de Saúde-SUS (DATASUS). A localização e mapa da da cidade de Manaus, com os limites de todos os bairros da cidade é apresentado na Figura 1,

A pesquisa realizada nesse artigo foi de cunho bibliográfico, a qual é definida por Severino (2017), como aquelas pesquisas que envolvem a análise de dados quantitativos e qualitativos simultaneamente. Onde as informações da análise quantitativa, onde o número de casos relacionados as variáveis meteorológicas e o custo do tratamento de doenças foram estimados para formar um estudo ecológico de série temporal, que se caracterizam por múltiplos eventos envolvendo variáveis específicas (doenças respiratórias), em um determinado período de tempo, através da base de dados meteorológicos do INMET.

Figura 1: Mapa mostrando a localização da área de estudo.

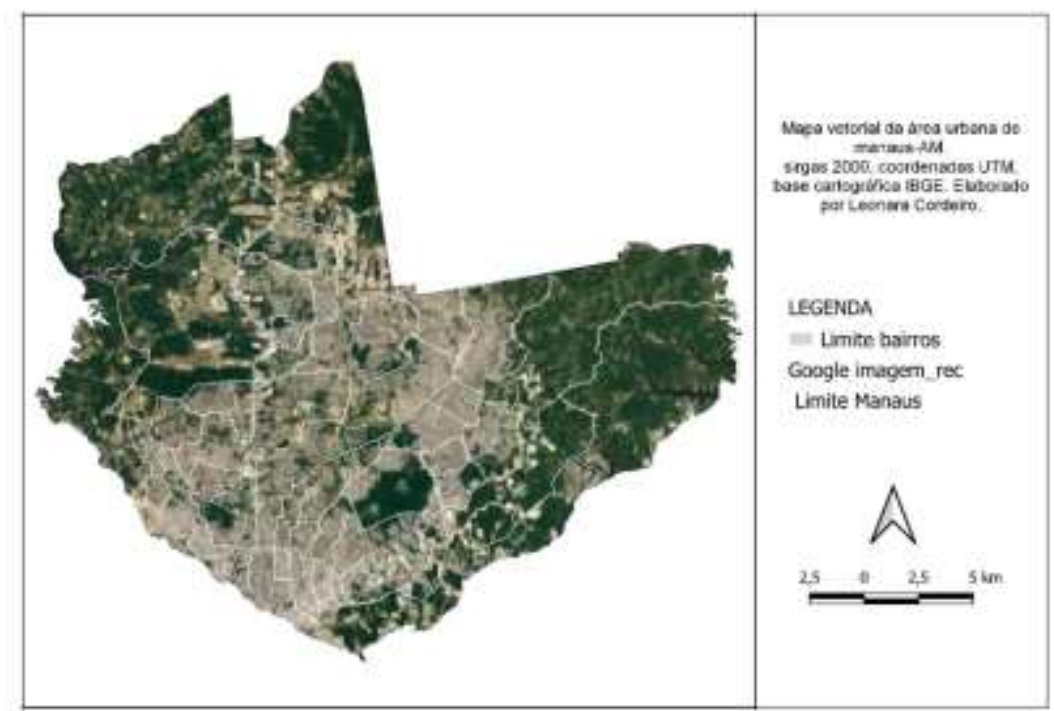

Fonte: Autores (2021).

Neste estudo, foi avaliada a relação entre o número de casos de doenças respiratórias hospitalizadas e o custo médio do tratamento com o SUS, e essas informações serviram de base para os dados meteorológicos (precipitação total anual, umidade relativa do ar e temperatura média). 
É usado uma variável que representa doenças causadas pelo sistema respiratório, classificadas pelo CID-10 como; faringite aguda, amigdalite aguda, laringite e ainda, traqueíte agudas e outras infecções agudas que afetam diretamente as vias aéreas superiores, como; a influenza (gripe), pneumonia, bronquite aguda e bronquiolite aguda, sinusite crônica, outras doenças do nariz e dos seios paranasais, doenças crônicas das amígdalas e das adenoides, outras doenças do trato respiratório superior, bronquite, enfisema e outras doenças pulmonares obstrutivas crônicas, asma, bronquiectasia, pneumoconiose, outras doenças do aparelho respiratório.

Os dados analisados referentes a internações por doenças respiratórias e os registros dos gastos referentes a doença repassados pela federação através do SUS. Além disso, foram coletados registros de internações e a doença diagnosticada justificando o atendimento nos hospitais. Mas apesar disso, devido a variabilidade temporal das informações coletadas, foram realizados somatórios anuais para que análise fosse realizada anualmente. Adcionalmente, foram utilizados os dados meteorológicos diários de temperatura média do ar $\left({ }^{\circ} \mathrm{C}\right)$, precipitação $(\mathrm{mm})$ e umidade relativa do ar (\%) da estação pertencente ao Instituto Nacional de Meteorologia (INMET), que no caso da precipitação são usadas o valor acumulado a cada ano, e as demais informações, temperatura $\left({ }^{\circ} \mathrm{C}\right)$ e umidade relativa $(\%)$ médias anuais foram obtidas.

\section{Resultados e Discussão}

A análise a seguir mostrará os registros das variáveis climáticas na cidade de Manaus/AM, durante o período de 1998 a 2020 e sua relação com os registros de doenças respiratórias, e os gastos monetários incorridos pelo SUS, para o tratamento dessa patologia.

A Figura 2 mostra os valores médios anuais que o SUS gastou anualmente com internações por doenças respiratórias, valores em torno de $\mathrm{R} \$ 19.048,28$. Nota-se que nos anos de 2003 a 2007 o total das internações se mantiveram-se altas e praticamente constante, sendo dispendido com o tratamento da doença um total anual acima de 14.000 (internados/ano), enquanto os recursos gastos com o tratamento da doença ficaram abaixo de 500 milhões de reais. Nos anos seguintes ocorreu um pequeno declínio no total de internações, apresentando totais entre 6.000 a 12.000 internados/ano, mas os recursos disponibilizados pela união para o tratamento da morbidade, que faz referência aos impactos econômicos com as internações por doenças respiratórias, podendo - se destacar os custos significativos na saúde pública em função do aumento da morbidade bem como dos custos de manutenção de equipamentos hospitalares, e provimentos de remédios. 
Figura 2: Total de casos de registros de doenças respiratórias (eixo da esquerda) e valor médio pago (SUS) pelas internações (eixo direita).

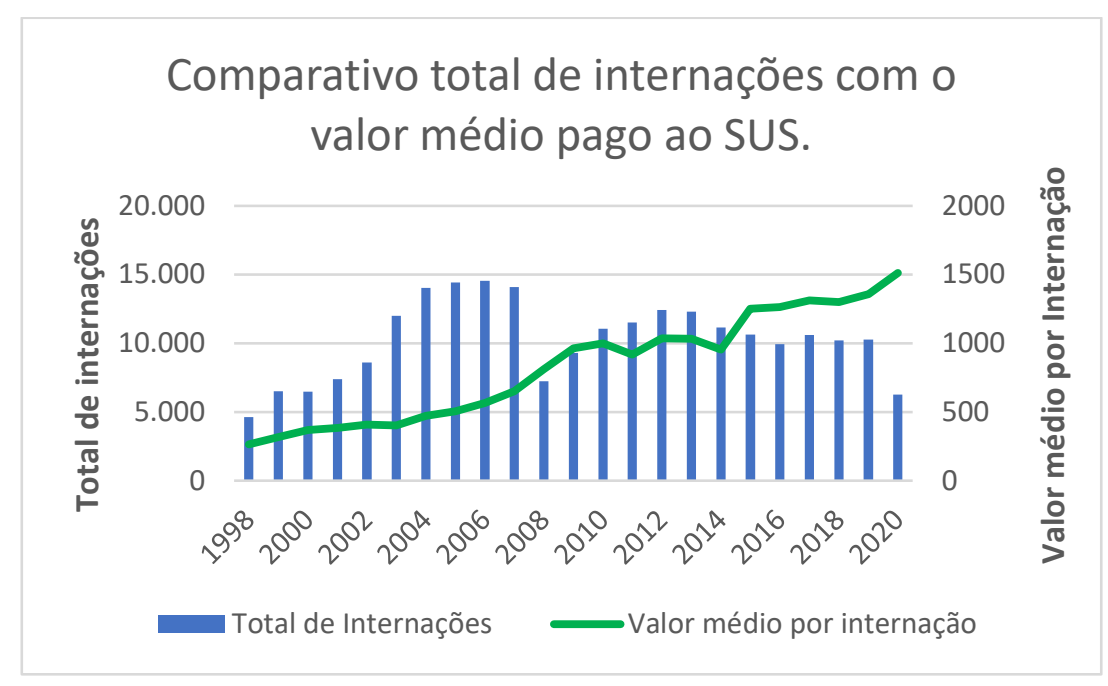

Fonte: Autores (2021).

Apesar do aumento dos recursos financeiros no tratamento da patologia e a diminuição nos casos durante aos primeiros anos analisados, as doenças do aparelho respiratório têm contribuído para o aumento do número de óbitos na cidade. Nota-se que em 1998 foram registrados 202 óbitos, que em relação a 2020, evidenciando um crescimento da curva para 688 óbitos/ano, número este que equivale a soma dos óbitos dos anos de 1999 e 2000 (Figura 3). Ainda, o aumento em 2020 está fortemente relacionado ao elevado número de focos de queimadas no estado. Segundo as informações do INPE, no ano de 2020, foram registrados durante os meses mais secos na região, o maior índice de focos ativos detectados na região, registrando 2.119 focos em julho, e alcançando em agosto a marca de 8.030 focos. Mas a questão é, o porquê o aumento no índice de doenças, o porquê o aumento de casos? Devido a ausência de informações da concentração de poluentes se faz necessário responder essa questão através do comportamento das variáveis meteorológicas, que associadas ao aumento das queimadas, contribuem para o aumento de casos de doenças cardiovasculares, infartos, agravamento de casos de asma, bronquiectasia, pneumoconiose (Aleixo \& Sant'Anna, 2014; Mandú et al., 2019; Carvalho et al., 2021; Santosnet al., 2021).

Figura 3: Total de casos de registros de internações por doenças respiratórias (eixo da esquerda), e o total de óbitos (eitxo da direita).

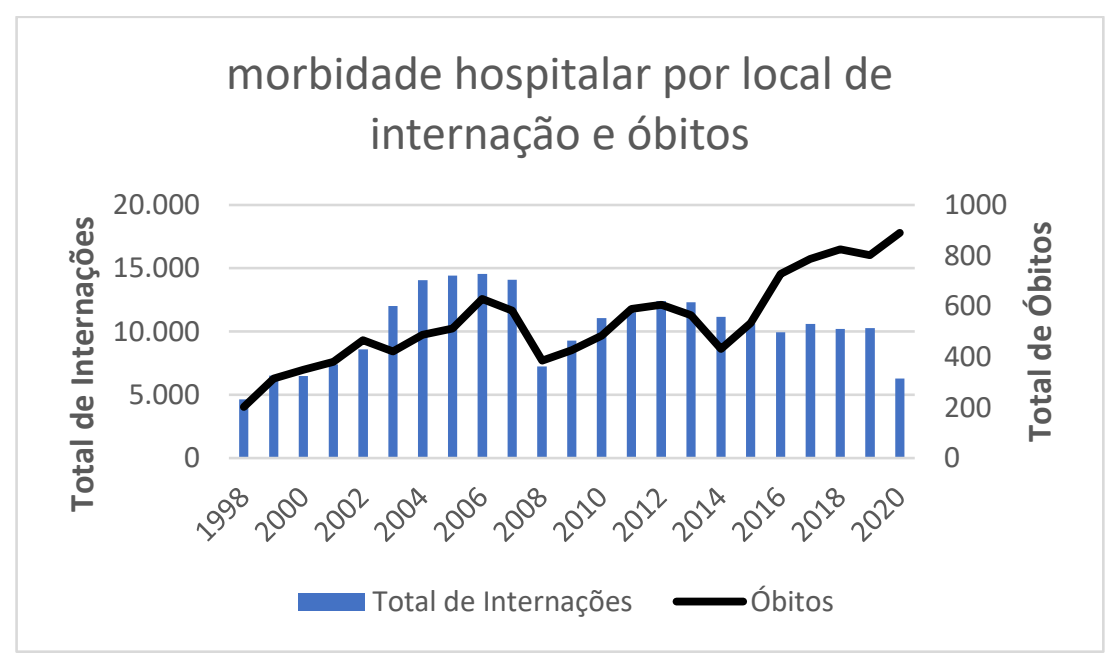

Fonte: Autores (2021). 
As variáveis climáticas afetam enormemente a saúde humana, e que associadas aos poluentes atmosféricos podem causar diversas morbidades. Mandú e Gomes (2019) Observou-se em Manaus um aumento gradativo do desconforto térmico humano e acredita-se que o aumento da incidência de doenças respiratórias está relacionado às condições climáticas. "O impacto na saúde, especialmente certas deteriorações patológicas causadas pelo comportamento de certos fatores climáticos da qualidade do ar, e a destruição da vitalidade urbana causada por certas precipitações extremas, será nossa ferramenta de recompensa. Afinal, não podemos estar imunes. O impacto das nossas ações sobre as consequências ambientais " (Monteiro, 1997, p.06).

Apesar do desconforto gerado pelas altas temperaturas na região, estas não foram as responsáveis pelo aumento das internações. Como podemos observar, durante os anos com maior incidência de internações (2003 a 2007) as temperaturas não ultrapassaram $28^{\circ} \mathrm{C}$. Ao contrário dos anos de 2014 a 2020 , temperaturas acima de $28,5^{\circ} \mathrm{C}$, anos em que as internações se mantêm em torno de 10.000 internações/ano, como mostra a Figura 4.

Figura 4: Total de casos de registros de internações por doenças respiratórias (eixo da esquerda), e temperatura média anual (eixo da direita).

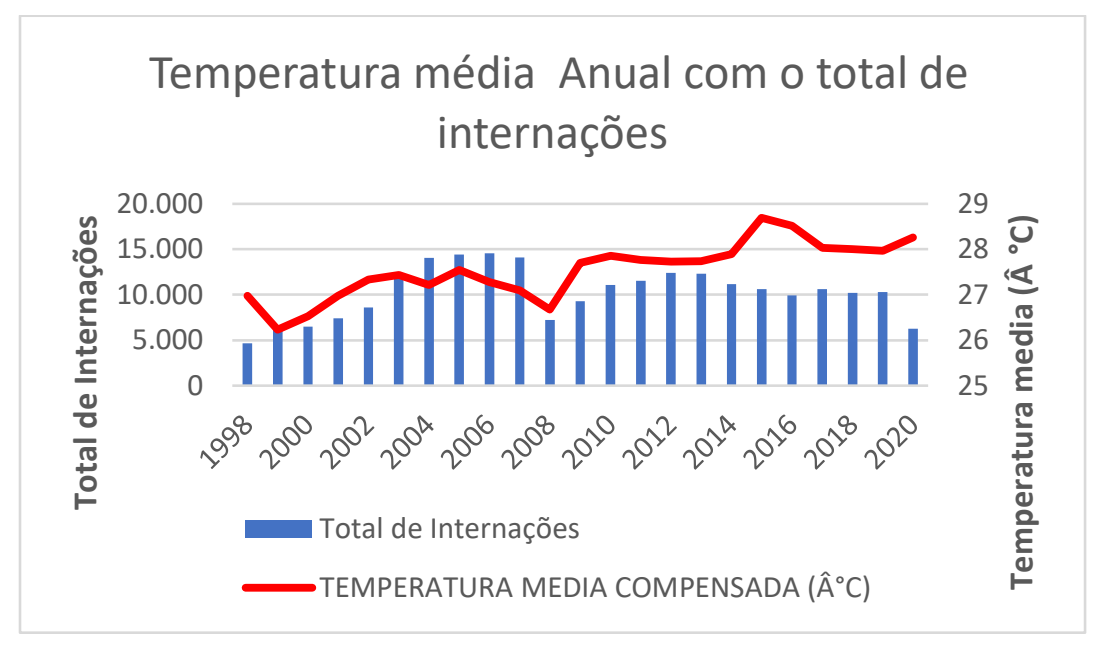

Fonte: Autores (2021).

As alterações climáticas ocorrem ao longo do tempo, e um dos motivos que levam a essa alteração, é o processo de urbanização, processo que vem acontecendo em Manaus a cada dia. Silva (2009) argumenta que o processo de urbanização pode alterar o clima em função de mudanças na superfície - uso e ocupação do solo - e aumentar no calor devido às mudanças no vento, umidade relativa do ar e precipitação. Um dos fatores importantes de serem analisados quando se analisa as doenças respiratórias é a umidade relativa (\%). Durante o período de 2010 a 2020 os registros são relativamente de anos secos em que são apresentadas baixo de $85 \%$ os períodos secos aumentam a ocorrência de problemas respiratórios. No ano de 2015 foi possível verificar umidades abaixo de $80 \%$.

No que se refere a umidade relativa do ar (ver Figura 5) mostram médias anuais em torno de 87\%, período que tem maior incidência de chuvas e $77 \%$ em períodos considerados secos, já em relação as temperaturas anuais são consideradas estáveis pois variam entre $26^{\circ} \mathrm{C}$ e $28^{\circ} \mathrm{C}$. 
Figura 5: Total de casos de registros de internações por doenças respiratórias (eixo da esquerda), e umidade relativa média anual (eixo da direita).

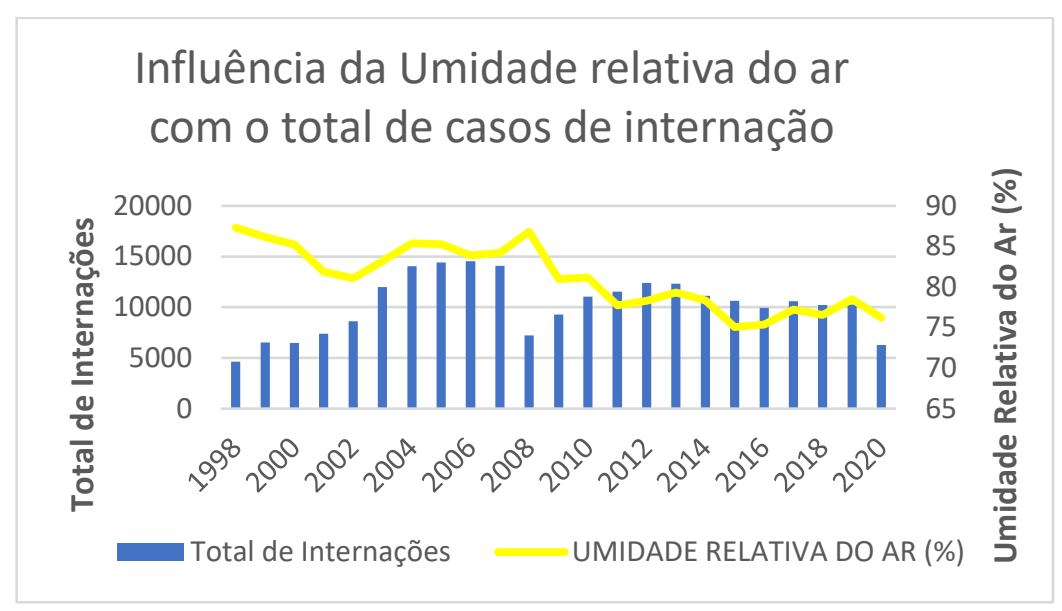

Fonte: Autores (2021).

A Figura 6 mostra a precipitação acumulada e o total internações por ano. Nota-se que nos anos de 1998, 1999 e 2000 existe precipitações acumulada a cada ano em torno de $2500 \mathrm{~mm}$, enquanto o número de internações ainda é baixo, ou seja, temos anos muito úmidos conforme observado pelo número de internações.

Ao contrário dos anos dos 2003, 2004, 2005, 2006 e 2007 que o número de internações excedera 14.000 internações/ano, enquanto a precipitação fica em torno $2.200 \mathrm{~mm}$. Já o ano de 2008 o número de internações é baixo, entorno de 7.237 internações/ano, quanto a precipitação acumulada ultrapassa $3.000 \mathrm{~mm}$. Com nessas informações, verifica-se que os anos que ocorrem muitas chuvas as internações diminuem.

Portanto a descrição dos volumes anuais, não apresentam um padrão homogêneo, visto que podem variar constantemente, influenciados não somente pelos sistemas atmosféricos regionais, mas também por eventos como o El Niño ou La Niña.

Figura 6: Total de casos de registros de internações por doenças respiratórias (eixo da esquerda), e umidade relativa média anual (eixo da direita).

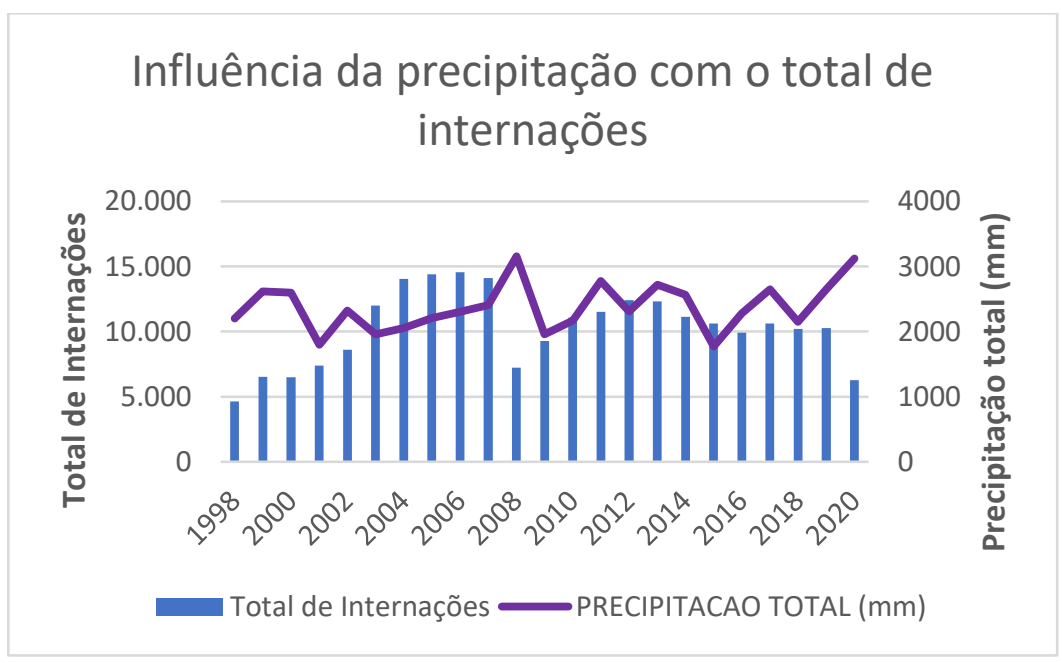

Fonte: Autores (2021). 
Conforme a análises dos dados meteorológicos a cidade de Manaus possui um clima predominante chuvoso, que ocorre de janeiro a abril e o mês de março apresentar maior precipitação (média de $310 \mathrm{~mm}$ ), tendo como períodos mais secos julho a setembro, porém agosto teve o índice mais seco com média de $(50 \mathrm{~mm})$, já os períodos de transição ocorrem nos meses maio, junho, outubro e dezembro pois chove em média 190 dias durante o ano, acumulando uma média anual de 3.000mm.

A análise dos resultados mostra que a relação entre os diversos atributos ambientais (temperatura média, umidade relativa, precipitação) e a relevância da taxa de hospitalização por doenças respiratórias, através da verificação dos dados, a temperatura é considerada um dos principais atributos ambientais que afetam doenças respiratórias. As doenças respiratórias pioram porque mudam ao longo do dia e afetam a imunidade do organismo. Por meio de mudanças climáticas extremas, ou seja, por meio dessas mudanças de quente para frio, o número de internações aumenta e, portanto, a taxa de mortalidade aumenta.

A baixa temperatura está em primeiro lugar relacionada com o declínio da capacidade vital e função, bem como a propagação de vários vírus, bactérias e alérgenos. Em certas condições, a temperatura já elevada irá reduzir a passagem de ar no trato respiratório e levar a maiores riscos. Portanto, as doenças respiratórias Hospitalização e mortalidade dos pacientes, os efeitos do frio e do calor se manifestam de diferentes maneiras no corpo. Levando em consideração as flutuações de temperatura, quanto mais baixa a temperatura, mais longo o efeito, e quanto mais alta a temperatura, mais curto o efeito e o efeito imediato.

A necessidade de proteger e preservar os recursos naturais voltados à reprodução e manutenção dos ciclos naturais reflete diretamente nas ações preventivas e corretivas da sociedade mundial. (PINA,2012). Assim, ao finalizar esse trabalho, percebe-se a necessidade de constante monitoramento dos elementos climáticos que afetam a cidade de Manaus, que mesmo rodeada de vegetação, não está isenta de mudanças climáticas em seu espaço ao longo do tempo.

\section{Conclusão}

Os resultados apresentados neste artigo concluíram que os dados climáticos e clínicos do Cid - 10 - doenças do aparelho respiratório está diretamente ligado aos números de internações, pois com a variação do clima entre calor e frio, afeta diretamente o sistema imunológico do ser humano, foram observados que eventos de o El Niño ou La Niña e aumento de queimadas, contribuem para o aumento dos casos e consequentemente mortalidade.

Neste estudo por indisponibilidade de informações não há dados referentes a tratamentos realizados em casa e atendimentos ambulatoriais e nem os custos de tratamento de doentes que não recorreram ao Sistema Único de Saúde, foi observado que as doenças do aparelho respiratório tem por sua vez um impacto significativo na economia não somente pelos gastos em saúde e assistência, mais por comprometer o crescimento econômico e o mercado de trabalho em razão da perda da capacidade laboral do ser humano.

Espera-se que os resultados aqui apresentados possam servir como um alerta ao governo junto administração sanitária da cidade de Manaus, a fim de chamar a atenção e promover incentivos para pesquisa de analises temporais pois a saúde não depende somente de fatores ambientais, necessitando pesquisa em outros fatores como (sanitárias, econômicas e sociais), que só através de incentivos haverá novos métodos de atenuação dos efeitos de chuvas nesses indivíduos, e para que sirva como alerta aos seres humanos que nossas ações ao meio ambiente não ficar imune pois a natureza reagir ao impactos que causamos a ela, por meio de suas alterações como fortes chuvas ou escassez ou temperaturas elevadas e nos sentimos as consequência por meio das doenças. Vale ressaltar que tais ações e as medidas teriam reflexos positivos consequentes em relação ao número de internações, o que irá reduzir gastos com o atendimento prestado, com medicamentos e outros serviços.

Como perspectivas para futuros trabalhos deseja-se analisar a influência das poluentes atmosféricas e as variáveis meteorológicas aos casos de morbidade ocorridos na cidade de Manaus. 


\section{Referências}

Aleixo, N. C. R. \& Sant'anna Neto, J. L. (2014). Condicionantes Climáticos e internações por pneumonia: estudo de caso em Ribeirão Preto/SP. Revista do Departamento de Geografia-USP, 27, 1-20.

Andreão, W.L.; Trindade, B.T.; Nascimento, A.P.; Júnior, N.C.R.; Andrade, M.F. \& Albuquerque, T.T.A. (2019). Influence of meteorology on fine particles concentration in Vitória Metropolitan region during wintestime. Revista Brasileira de Meteorologia, 34 (4), $459-470$.

Anjos, I. B. \& Ferreira, M. E. M. C. (2011). Estudo das Internações por Doenças Respiratórias e a Variabilidade Climática em Maringá-Paraná. Geoingá: Revista do Programa de Pós-Graduação em Geografia Maringá, 3 (2), 56-81.

Artaxo, P.; Gatti, L. V.; Leal, A. M. C.; Longo, K. M.; Freitas, S. R.; Lara, L. L.; Pauliquevis, T. M.; Procópio, A. S. \& Rizzo, L. V. (2005). Química atmosférica na Amazônia: a floresta e as emissões de queimadas controlando a composição da atmosfera amazônica. Acta Amazônica. 35, 185-196.

Barcellos, C.; Monteiro, A. M. V.; Corvalán, C.; Gurgel, H.; Carvalho, M. S.; Artaxo, P.; Hacon, S. \& Ragoni, V. (2009). Mudanças climáticas e ambientais: cenários e incertezas. Epidemiologia Serviços de Saúde, Brasília, 18 (3): 285-304.

Barcellos, C. \& Hacon. S. S. (2016). Um grau e meio. E daí? Caderno de Saúde Publica, 32 (3):e00212315.

Bruzos, G. A. de S.; Kamimura, H. M.; Rocha, S. A.; Jorgetto, T. A. C.; Patrício, K. P. Meio Ambiente e Enfermagem: suas interfaces e inserção no ensino de graduação. Saúde \& Sociedade, v.20, n.2, 2011, pp.462-469.

Carvalho, E. K. M. A.; Dantas, R. T. \& Carvalho, J. R. M. (2021). Influência de variáveis Meteorológicas na ocorrência de pneumonia. Gestão \& Regionalidade, 37 (110), 287-304.

Departamento de Informática do Sistema Único de Saúde. Informações de Saúde. Indicadores de Saúde [Internet]. Morbidade Hospitalar por residência da CID10. http://datasus.gov.br

Dutra, J.; Lima, A. A.; Canalez, G. \& Lopes, M. (2021). Predições de qualidade do ar com base nas estimativas de emissões de gases de efeito local do sistema de transportes de Manaus, Amazonas. Revista Ibero-Americana de Ciências Ambientais, 12 (4).

Freitas S.R. Longo, K. M.; Dias, M. A. F.; Dias, P. L. S.; Chatfield, R.; Prins, E.; Artaxo, P.; Grell, G. A. \& Recuero, F. S. (2005). Monitoring the transport of biomass burning emissions in South America. Environmental Fluid Mechanics, 5 (1-2), 135-167.

Instituto Nacional de Pesquisas Espaciais (INPE). (2008). Monitoramento da cobertura florestal da Amazônia por satélites Sistemas PRODES, DETER, DEGRAD e QUIEIMADAS 2007-2008, report, Coord. Geral de Obs. da Terra, Minist. da Ciência e Tecnol., São José dos Campos, Brasil. (ww.obt.inpe.br/prodes/Relatorio_Prodes2008.pdf)

Magalhães, E. S.; Silva, L. M. G. F.; Lima, A. A.; Dutra, G. S. S.; Bezerra, I. F. O. \& Costa, P. S. (2019) The influence of meteorological variables and air pollutants on the occurrence of respiratory diseases in the city of Manaus/AM. International Journal of Development Research, 9 (12), $32193-32197$.

McMichael, A. J. (1999). From hazard to habitat: Rethinking environment and health. Epidemiology,10 (4), $460-464$.

McMichael, A. J. (2003). Global climate change and health: an old story writ large. In: Mcmichael, A.J. et al. (eds). Climate change and human health. Risks and responses. Genebra: WHO, 1-17.

Mandú, T. B.; Gomes, A. C. S.; Vale, R. S. \& Santos, M. S. (2019). Associação entre o índice de calor e internações por infarto agudo do miocárdio em ManausAM. Hygeia 15 (31): 16-28.

Monteiro, C. A. F. (1976). O clima e a organização do espaço no Estado de São Paulo: problemas e perspectivas. São Paulo: IGEOG/USP, n 28, Série “Teses e Monografias".

Monteiro, C. A. F. (2003). Teoria e clima urbano. In: Clima urbano. São Paulo: Contexto, 09-68.

Moraes, D. S. L. \& Jordão, B. Q. (2002). Degradação de recursos e seus efeitos sobre a saúde humana. Revista de Saúde Publica, 36 (3), 370 -374.

Moreno, A.R. (2006). Climate change and human health in Latin America: drives, effects, and policies. Environmental Change, 6, $157-164$.

Pina, J. H. A. (2012). A influência das áreas verdes urbanas na qualidade de vida: O caso dos Parques do Sabiá e Victório Siquierolli em Uberlândia-MG. Ateliê Geográfico, 6 (1), 143-169.

Pitton, S. E. \& Domingos, A. E. (2004). Tempos e doenças: efeitos dos parâmetros climáticos nas crises hipertensivas nos moradores de Santa Gertrudes -SP. Estudos Geográficos. Rio Claro, 2 (1), 75-86.

Santos, M. R. P.; Silva, W. C.; Silva, E. B. R. \& Gomes, A. C. S. (2021). Dinâmica espaço-temporal: condições atmosféricas versus manifestações de doenças respiratórias no Baixo Amazonas e Sudoeste do Pará. Revista Brasileira de Geografia Física, 14 (5), $2790-2805$.

Serra,S. R. \& Mülfarth, R. C. K. (2021). Impactos das queimadas da região centro-oeste do Brasil sobre as cidades do estado de São Paulo. Brazilian Journal of Development, 7 (5), 51237-51257.

Severino, A.J. (2017). Metodologia do trabalho científico, livro eletrônico,(2a ed.),Cortez

Silva, A. A. M. A. (2012). Reflexões sobre o conceito de clima e alterações climáticas: uma reflexão ou equívoco ¿. Revista Geonorte, Edição Especial, 2 (4), $1048-1061$. 
Research, Society and Development, v. 10, n. 14, e98101421841, 2021

(CC BY 4.0) | ISSN 2525-3409 | DOI: http://dx.doi.org/10.33448/rsd-v10i14.21841

Silva, D. A. (2009). A influência das áreas verdes no clima da cidade de Manaus. Dissertação de Mestrado, Universidade Federal do Amazonas, Manaus, Amazonas. 293 pp.

Sousa, T. C. M.; Barcellos, C. B.; Gracie, R.; Hacon, S. Covid-19 e queimadas na Amazônia Legal e no Pantanal: aspectos cumulativos e vulnerabilidades. Nota Técnica Instituto de Comunicação e Informação Científica e Tecnológica em Saúde (ICICT) Fundação Oswaldo Cruz (Fiocruz), 2021.

Torres, T. L. \& Medeiros, A. P. P. (2019). Internações por doenças do aparelho circulatório e a queima de biomassa florestal em residentes de Porto Velho Rondônia entre 2014 -2015. Saber Científico, 8 (1), 1-8. 